Reference for citation please: ALHAJJ MA., BOURGUIGNON S., PALMA LOPES S., VILLAIN G., Joint inversion of electromagnetic measurements for the determination of water saturation profiles in concrete structures, Cement and Concrete Research, Vol. 147, September 2021. https://doi.org/10.1016/j.cemconres.2021.106500

\title{
Joint inversion of electromagnetic measurements for the determination of water saturation profiles in concrete structures
}

\author{
Marie Antoinette ALHAJJ ${ }^{a, *}$, Sébastien BOURGUIGNON ${ }^{b}$, Sérgio PALMA-LOPES ${ }^{c}$ and \\ Géraldine VILLAIN ${ }^{a}$
}

${ }^{a}$ MAST-LAMES, Université Gustave Eiffel, Campus de Nantes, 44344 Bouguenais, France
${ }^{b}$ LS2N, Centrale Nantes, 44321 Nantes, France
${ }^{c}$ GERS-GeoEND, Université Gustave Eiffel, Campus de Nantes, 44344 Bouguenais, France

\section{ARTICLE INFO}

\section{Keywords:}

Durability of concrete

Non destructive evaluation techniques

Electromagnetic properties

Joint inversion

\begin{abstract}
A B S TRACT
Water saturation profiles in concrete are essential to assess its durability and can be determined using non-destructive techniques, especially the electric and the capacitive methods. In this paper, we propose a new inversion scheme where both resistivity and permittivity measurements are inverted jointly to retrieve the saturation profile. The finite element method is used to model the measurements in 3D, the concrete having a saturation profile with depth, represented by a continuous model taking the form of a Weibull curve with four parameters. A non-linear leastsquares optimization based on the Levenberg-Marquardt scheme is developed for the inversion of measurements. Results show that information gathered from both measurements enriches the reconstructed profile, leading to a more reliable estimation of saturation profiles. We believe that the joint inversion method herein developed could lead to the study of more complex phenomena, such as the coupled water-chloride ingress.
\end{abstract}

\section{Introduction}

Innovative construction technologies are interested in preserving the durability of concrete to extend the service life of a structure. Many studies have shown that non-destructive testing (NDT) methods are useful and offer many advantages, being completely harmless to the structure and providing monitoring through measurements that can be performed repeatedly. Therefore, with the improvement of numerical methods to analyze these measurements, NDT methods are promising in terms of durability assessment $[1,2,3]$. Among the existing NDT methods, this paper focuses on the electrical method (measuring the apparent resistivity) and on the capacitive method (measuring the apparent dielectric permittivity). The measurements are called apparent because they represent integrating values from the concrete surface to a certain depth that depend on the electrode size and spacing [4].

Previous studies have shown that the electrical resistivity, $\rho$, is sensitive to the change of concrete saturation degree, $S$, which is a prominent durability indicator $[5,6,7]$. This sensitivity could be established by an experimental calibration law between $\rho$ and $S[8,9,10,11]$. Nonetheless, an inversion of the measurements needs to be carried out to estimate the resistivity of concrete. The measurements using the electric method are apparent values that depend on the electrode configuration and geometry used, hence on the investigation volume of the measurements [12, 13, 14]. Determining the water content profile with this technique was already proposed in the literature [15, 16,17], consisting of three steps. First, electric measurements are performed on the structure. Then, the measurements are inverted to estimate the electric resistivity profile in concrete. Finally, $\rho$ is converted into $S$ using an experimental calibration performed independently. However, the electric method has limitations. For instance, resistivity measurements in a too dry medium are very noisy, thus unreliable $[18,13,19,20,2]$. The range of resistivity values highly depends on environmental factors and the concrete composition, varying from $50 \Omega . m$ for very wet concrete to more than $4000 \Omega . m$ for dry concrete [21].

Similarly, the dielectric permittivity, $\varepsilon$, is sensitive to the change of concrete saturation degree [22, 23, 24]. This sensitivity could be expressed by an experimental calibration law between $\varepsilon$ and $S$ [25]. Capacitive measurements need to be inverted to estimate the dielectric permittivity profile of concrete [26]. Then, $\varepsilon$ is converted into $S$ using

\footnotetext{
${ }^{*}$ Corresponding author

@marie-antoinette.alhajj@univ-eiffel.fr (M.A. ALHAJJ); sebastien.bourguignon@ec-nantes.fr (S. BOURGUIGNON); sergio.palma-lopes@univ-eiffel.fr (S. PALMA-LOPES); geraldine.villain@univ-eiffel.fr (G. VILLAIN)

ORCID(s): 0000-0001-7711-5103 (M.A. ALHAJJ); 0000-0002-6660-2704 (S. BOURGUIGNON); 0000-0001-7022-389X (S.

PALMA-LOPES); 0000-0002-4478-034X (G. VILLAIN)
} 
experimental calibration. However, all the effects due to the building of the concrete structure, such as voids due to air bubbles, can lead to important errors in the measured apparent permittivity [21]. Furthermore, the number of collected data if often small, and sometimes insufficient to determine precisely the saturation distribution in the whole medium.

Studies based on the inversion of data in geophysics have shown that the joint inversion of different NDT methods has many benefits [27, 28, 29]. First, the number of collected data could increase significantly, leading to a better assessment of the soil property. Second, combining NDT methods that are all sensitive and in a different way to a particular property enriches the information gathered from measurements and can increase the estimation accuracy [30, 31, 32, 33, 34]. For concrete structures, several authors recommended combining various NDT methods [35, 36]. This combination of techniques consists of a combination of independent sets of results. Measurements from different NDT methods are gathered and combined to predict a certain concrete property without combining them in the inversion procedure and without considering gradients in the dimensions of the concrete structure. Therefore, this paper presents a new methodology that combines the two electromagnetic techniques, by performing a joint inversion procedure, to determine the water saturation degree distribution in concrete. Both measurements being sensitive to saturation but differently, the information gathered may yield to a better prediction accuracy of the saturation profile.

To perform the inversion of measured electromagnetic data, we first study the forward problem, that consists of numerically simulating apparent resistivity and permittivity measurements on concrete for a given saturation profile. Then, we build an inversion scheme based on the minimization of a least-squares cost function jointly defined on resistivity and permittivity data. The joint inversion scheme is first tested on synthetic data. Numerical experiments using noise-free and noisy data are conducted to study the performance of the joint inversion procedure, compared to the separate inversion of the two data sets. Finally, the joint inversion scheme is tested on experimental data collected from laboratory measurements.

The assumptions adopted to carry out this study are briefly stated in the following. Firstly, to better constrain the problem, we consider a parametric model represented by a continuous function to describe the saturation degree. Secondly, the present work aims at dealing with the phenomena of drying or imbibing of concrete, considered non-cyclic and unidirectional. Therefore, we will consider a monotonic curve to describe concrete saturation degree throughout the paper as it was justified in [26]. The saturation profile is represented by a Weibull distribution, involving the estimation of four parameters. Thirdly, we are only interested in the distribution of the saturation degree with depth considering that concrete is homogeneous regarding the two other dimensions. Nonetheless, a 3D concrete model is required to compute apparent data using the finite element modelling (FEM) method.

The remainder of this paper is organized as follows. An overview of the two electromagnetic properties of concrete and their measurement methods is presented in Section 2. In Section 3, the inversion methodology for the estimation of the saturation profile from resistivity and permittivity data separately is first presented. Then, the joint inversion algorithm is detailed. The joint inversion method is then validated on numerical simulations, and sensitivity studies are discussed in Section 4. An experimental study on drying concrete is presented in Section 5, where electrical and capacitive measurements are inverted separately and jointly. The results are discussed in Section 6 and the conclusion in Section 7 closes the paper.

\section{Electromagnetic and hydric properties of concrete}

In this section, a brief definition is provided for both electrical resistivity and dielectric permittivity. The measuring principles for both quantities, as well as the corresponding electrode configurations used in this study, are presented. Then, the water saturation degree of concrete and its sensitivity to electromagnetic properties are reviewed.

\subsection{Electrical resistivity}

\subsubsection{Definition}

The DC (direct current) electrical resistivity of a material, noted $\rho(\Omega . m)$, is the ability of the material to oppose the flow of free electric charges when it is subjected to an electric field [37]. In electrostatics, Poisson's equation is given by Eq. (1) and links the electric potential scalar field $V$ to a given resistivity distribution:

$$
\nabla \cdot\left(\frac{1}{\rho} \nabla V\right)=0
$$

where $\nabla \cdot$ is the divergence operator and $\nabla$ the gradient operator. 


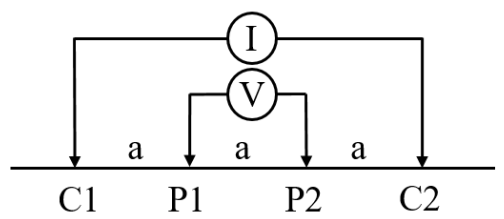

(a)

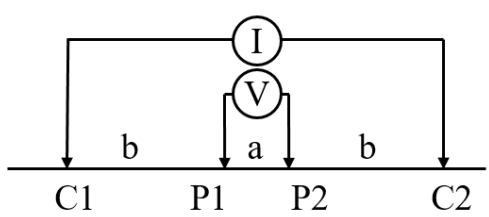

(b)

Figure 1: Scheme of electrode configurations used in this study: (a) the Wenner alpha array (b) the Schlumberger array.

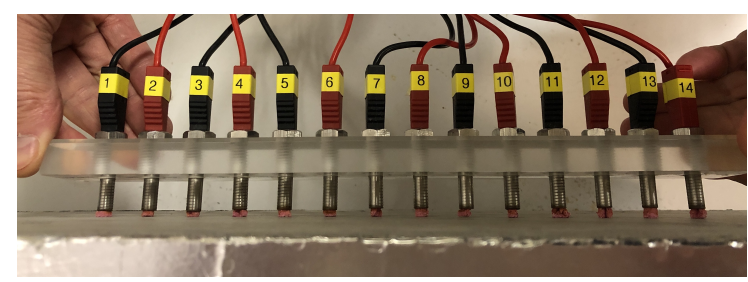

Figure 2: A 14-electrode probe for electrical resistivity measurements.

\subsubsection{Measurement principle and device}

The resistivity measurement principle consists of applying a direct current on a pair of electrodes and measuring the potential drop between other pairs of electrodes. There are many possible electrode combinations to perform the measurements. In this study, we are interested in two 4-electrode configurations, the Wenner alpha configuration (simply designated as Wenner throughout the paper) with 4 equally spaced electrodes and the Schlumberger configuration with unequally spaced electrodes (Fig. 1) [38].

The measuring device consists of a multi-electrode resistivity probe developed by Du Plooy et al. (2013) [7] for electrical resistivity measurements, which is shown in Figure 2. The probe is made of 14 electrodes that are equally spaced with a 20-mm interval. Using this device, the resistivity distribution with depth in concrete can be determined by making measurements on the surface, using different spacings between electrodes. Using all possible values for electrode spacing, a total number of 100 apparent resistivity values can be collected for each measuring sequence, which go deeper into concrete's depth when the electrode spacing is larger.

The measured apparent resistivity $\rho_{a}(\Omega . m)$ is defined as the ratio of the measured voltage $V(V)$ to the applied current intensity $I(A)$, multiplied by a geometric constant $G_{R}(m)$ :

$\rho_{a}=G_{R} \frac{V}{I}$

where the geometric factor $G_{R}$ depends on the inter-electrode spacings, on the position of the resistivity probe and the geometry of the structure [39]. In this paper, $G_{R}$ is computed by numerical modeling using the finite element software COMSOL Multiphysics®.

In our case, where we specifically investigate inhomogeneity of the water content (and thus of the resistivity), the measured resistivities only provide apparent resistivity values, which need to be inverted to estimate the resistivity distribution in the concrete medium. This question is addressed in Section 3.3.

\subsection{Dielectric permittivity}

\subsubsection{Definition}

The dielectric permittivity, noted $\varepsilon(F / m)$, is related to the phenomenon of electric polarization resulting from the relative displacement of the bound charges of the material under the action of an external electric field [21]. Due to the strong dependence of the polarization phenomenon on the oscillation frequency of the electric field and the energy losses that accompany that phenomenon, the permittivity is expressed as a complex quantity, where the real part takes into account the polarization effect and the imaginary part describes the energy losses [40].

As mentioned in [26], the capacitance is the ability of the material to store electric energy and it depends on the real part of the complex permittivity. Therefore, the dielectric constant of concrete, which will be noted $\varepsilon$ throughout the 


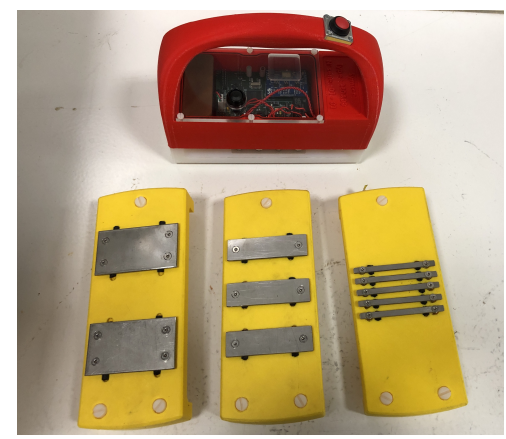

Figure 3: Capacitive measurement system with the four different electrode arrays. Yellow arrays from left to right are LE1, ME1, SE1 and white array connected to the red system is SE2 (see text for details).

paper to simplify notations, is actually equal to the real part of the relative complex permittivity.

Electromagnetic problems are expressed using Maxwell equations. However, these equations can be simplified for the capacitive problem using the electrostatic approximation [26], then reducing the problem to the following equation:

$\nabla \cdot \varepsilon_{0} \varepsilon \nabla \varphi=0$

where $\varphi$ is the spatial distribution of the electric potential and $\varepsilon_{0}$ is the vacuum permittivity.

\subsubsection{Measurement principle and device}

The permittivity measurement principle consists of applying an alternating current between two or more electrodes placed on the surface of the sample, and then measuring the resonance frequency of the electrode-concrete system which acts as an oscillating circuit. The air-concrete system forms a capacitor, whose capacitance $C$ essentially depends on the geometry of the electrodes and the dielectric properties of the medium underneath.

The measuring device used in this paper and shown in Figure 3 was developed at Universite Gustave Eiffel and is based on several configurations corresponding to four different types of probes, consisting of metal electrodes inserted in a Plexiglas ${ }^{\circledR}$ support. The probes differ by their number of metal plates forming the electrodes and by their dimensions, which make their investigation depths different [26]. More precisely, it is composed of:

- LE1, a capacitive array consisting of 2 plates of $70 \times 40 \mathrm{~mm}^{2}$, with a spacing between the plates equal to $40 \mathrm{~mm}$,

- ME1, a capacitive array consisting of 3 plates $70 \times 15 \mathrm{~mm}^{2}$ with a spacing between the plates equal to $15 \mathrm{~mm}$,

- SE2, a capacitive array consisting of 4 plates $70 \times 10 \mathrm{~mm}^{2}$ with a spacing between the plates equal to $10 \mathrm{~mm}$,

- SE1, a capacitive array consisting of 5 plates $70 \times 5 \mathrm{~mm}^{2}$ with a spacing between the plates equal to $5 \mathrm{~mm}$.

Using this device, the apparent dielectric permittivity in concrete can be determined by making measurements on the surface, using the capacitive arrays listed above. A total number of four apparent permittivity values can then be collected for each measuring sequence. The larger the electrode dimensions and spacing, the larger the depth of investigation.

When placed on a material with a given permittivity, the capacitive device measures the resonance frequency of the system, which will correspond to a certain capacitance and dielectric permittivity. Therefore, performing these measurements on different materials with known permittivity, a relation between the resonance frequency and the dielectric permittivity can be established for each capacitive array, as given by Eq. (4) [26]. The resonance frequency for any unknown material can be translated into an apparent permittivity value by:

$\frac{1}{f_{\text {air }}^{2}}-\frac{1}{f_{\text {mat }}^{2}}=A \varepsilon_{a}+B$

where $f_{\text {air }}$ is the resonance frequency in air, $f_{\text {mat }}$ the resonance frequency in the material, $A$ and $B$ two constants that depend on the dimensions of the capacitive probe. 
The apparent permittivity $\varepsilon_{a}$ obtained by Eq. (4) equals the permittivity of concrete in the case of a homogeneous medium. However, similarly to the resistivity measurements discussed in Section 2.1.2, such measurements only provide apparent permittivity values in the case of inhomogeneous water content. Their inversion will be discussed in Section 3.3.

\subsection{Concrete saturation degree}

The degree of saturation, noted $S$, represents the fraction of the volume occupied by water $\left(V_{w}\right.$ in $\left.\mathrm{m}^{3}\right)$ in the total pore volume $\left(V_{v}\right.$ in $\left.\mathrm{m}^{3}\right)$ :

$S=\frac{V_{w}}{V_{v}}$

The value of $S$ conditions the penetration of aggressive agents [41, 42, 43, 44, 45, 46, 47]. Therefore, the determination of the saturation degree contributes to predicting the degradation of concrete. Both electromagnetic properties of concrete considered in this paper ( $\rho$ and $\varepsilon$ ) are sensitive to concrete saturation degree. This relation results in experimental laws, which are determined by calibration on test specimens. The electrical resistivity $\rho$ was shown to be exponentially related to $S$ :

$\rho=A_{R} S^{-B_{R}}$

with $A_{R}, B_{R}$ two positive constants. Eq. (6) is consistent with Archie's empirical law, proposed in 1942 by geologists in the oil prospecting field for non-clayey rocks [48], and then for porous materials such as concrete [9, 10, 49, 11]. The dielectric permittivity $\varepsilon$ was shown to increase linearly with $S[50,51,25]$ :

$\varepsilon=A_{P} S+B_{P}$

with $A_{P}, B_{P}$ two positive constants. The calibration of $\rho$ and $\varepsilon$ (that is, the determination of the values of constants $A_{R}$, $B_{R}, A_{P}$ and $B_{P}$ ) is valid for a given concrete formulation.

\section{Methodology}

In this section, the methodology for estimating the concrete saturation degree profile is presented. First, a model for the saturation degree profile is described. Then, the computation of the forward problems, predicting the values of each electromagnetic quantity for a given saturation profile, is explained. Finally, our joint inversion procedure is proposed.

\subsection{Saturation degree profile}

This study deals with the phenomenon of drying or imbibing concrete, considered non-cyclic and unidirectional, by estimating the water saturation degree of concrete as a function of depth at a given time interval. That is, the degree of saturation in concrete is assumed to depend only on the depth $z$ inside the structure, and a monotonically increasing (respectively decreasing) profile is assumed when drying (respectively imbibing) is considered.

Estimating such depth profile from surface measurements is a difficult issue, in particular, because the number of data is usually small and the sensitivity of the measurements to the saturation degree gets weaker as the depth increases. Therefore, the searched saturation profile has to be constrained. In this paper, we follow the choice made in previous studies, where the saturation profile was shown to be adequately adjusted with the following Weibull curve expression $[52,26]$ :

$S=\left(\theta_{1}-\theta_{2}\right) \exp \left(-\left(\frac{z}{\theta_{3}}\right)^{\theta_{4}}\right)+\theta_{2}$

where $z$ is the depth in concrete $(m), \theta_{1}$ is the saturation degree of concrete at the surface $(\%), \theta_{2}$ is the asymptotic saturation degree at infinite depth (\%), $\theta_{3}$ is the scale parameter of the curve $(m)$ and the unit-free parameter $\theta_{4}>0$ is the shape parameter of the curve.

This model can represent both drying and imbibing cases. Note that, alternately, previous studies considered a multi-layer discrete model [51, 53]. This model relies on the preliminary definition of the number of layers and their corresponding thicknesses. Then, the saturation degree associated with each layer is estimated. This model is more 
flexible and can represent a wider variety of profiles, in particular non-monotonic ones. In our case, however, we focus on drying and imbibing cases, which are both monotonic. Therefore, we prefer the Weibull model in Eq. (8), essentially because it is more constrained, which may be more suited in environments with noisy data. It also requires a smaller number of parameters to be estimated, which is also important from a numerical point of view. Finally, note that other continuous models could be considered. For example, the arctangent or sigmoid functions, which have a similar shape to that of the Weibull model, could also accurately represent the saturation degree profile. Additional parameters could be similarly incorporated in order to account for the bounds of the profile (values at both extremities of the inspected piece) and its corresponding shape and scale.

\subsection{Forward problem}

We now describe the forward problem, that allows the computation of the apparent resistivities $\rho_{a}$ and the apparent permittivities $\varepsilon_{a}[50,54,51,26,55]$ at the measurement points. This problem is governed by Eq. (1) and Eq. (3) respectively and consists of solving a set of partial differential equations. Apart from trivial cases, we generally do not have an analytical solution to the equation. For that, we consider the finite element method, a numerical method used to numerically compute an approximate solution $[56,57]$. For this purpose, the commercial finite element modeling software COMSOL Multiphysics ${ }^{\circledR}$ (v.5.4) is used and the concrete model is defined: geometry, mesh, electrode position for both electrical and capacitive problems. Concerning the geometry, a block of dimensions $500 \times 250 \times 120$ $\mathrm{mm}^{3}$ is used to model the concrete slab. The block dimensions are identical to the ones that will be used in the experimental study (Section 5). 3D tetrahedral elements with quadratic shape functions are used to model measurements accurately, elements in the vicinity of the electrodes (of resistivity and capacity) have a maximum size equal to $a / 10$, $a$ being the spacing between two consecutive resistivity point electrodes, and $a$ being the smallest electrode length of a capacitive array. Meshes of gradually larger dimensions are used in the rest of the domain such that the forward problem computation time is about $1 \mathrm{~min} 30 \mathrm{~s}$. Then the saturation profile is defined by the Weibull curve in Eq. (8).

For the electric problem, the multi-electrode resistivity probe (Section 2.1.2) is represented by 14-point electrodes (see Fig. 10(a) in Section 4). Knowing the saturation depth profile, the resistivity depth profile is obtained using the calibration relation in Eq. (6). Then, the COMSOL Multiphysics module AC/DC Electric Currents is used to compute the apparent resistivities $\left(\rho_{a_{m o d}}\right)$ by solving Eq. (1) and Eq. (2). A positive intensity current is injected through one electrode, a negative intensity current through another and an electric potential is simulated at the remaining electrodes. A total number of 100 apparent resistivity measurements are computed.

For the capacitive problem, the capacitive plates (Section 2.2.2) are represented by surface plates with a negligible thickness (Fig. 10(b)). For a given saturation depth profile, the permittivity depth profile is obtained using the calibration relation in Eq. (7). The COMSOL Multiphysics module AC/DC Electrostatics is used to compute the apparent permittivities by solving Eq. (3). First, the capacitance due to the applied permittivity profile is computed then apparent permittivities $\left(\varepsilon_{a_{\text {mod }}}\right)$ are obtained, for each capacitive array (LE1, ME1, SE2 and SE1).

For both DC-electric and capacitive models, the following conditions are considered: current conservation within the concrete block, electric insulation of the block boundaries and a null initial potential in the block. Note that other electrode configurations could be considered, especially to increase the number of permittivity measurements compared to that of the resistivity measurements, yet only the electrode configurations that are accessible with the available experimental devices (Section 2) are numerically simulated.

\subsection{Inversion procedure}

In this section, we describe a method to invert apparent resistivities $\rho_{a}$ (respectively, apparent permittivities $\varepsilon_{a}$ ) and to retrieve the concrete properties ( $\rho$ and $\varepsilon$ respectively). It is based on minimizing the least-squares misfit criterion:

$\psi(\boldsymbol{\theta})=\sum_{i=1}^{n}\left(d_{i_{\text {mod }}}(\boldsymbol{\theta})-d_{i_{\text {mes }}}\right)^{2}$

where $\mathbf{d}_{\text {mod }}(\boldsymbol{\theta})$ is the vector collecting each simulated data point $d_{i_{\text {mod }}}(\boldsymbol{\theta})$ for a given set of profile parameters in vector $\boldsymbol{\theta}=\left[\theta_{1}, \theta_{2}, \theta_{3}, \theta_{4}\right]$ and $\mathbf{d}_{\text {mes }}$ similarly collects the measured data. We propose to minimize the function $\psi$ using the Levenberg-Marquardt nonlinear least-squares procedure [58, 59]. The inversion scheme is explained in Fig. 4. It is an iterative process which starts with an initial saturation profile given by initial parameters $\boldsymbol{\theta}^{(0)}$. Using calibration curves given in Eq. (6) and Eq. (7), the corresponding resistivity/permittivity profile is obtained. Then the initial data prediction $\mathbf{d}_{\text {mod }}^{(0)}$ is computed in the forward problem and the criterion $\psi\left(\boldsymbol{\theta}^{(0)}\right)$ is computed. Initial parameters are 


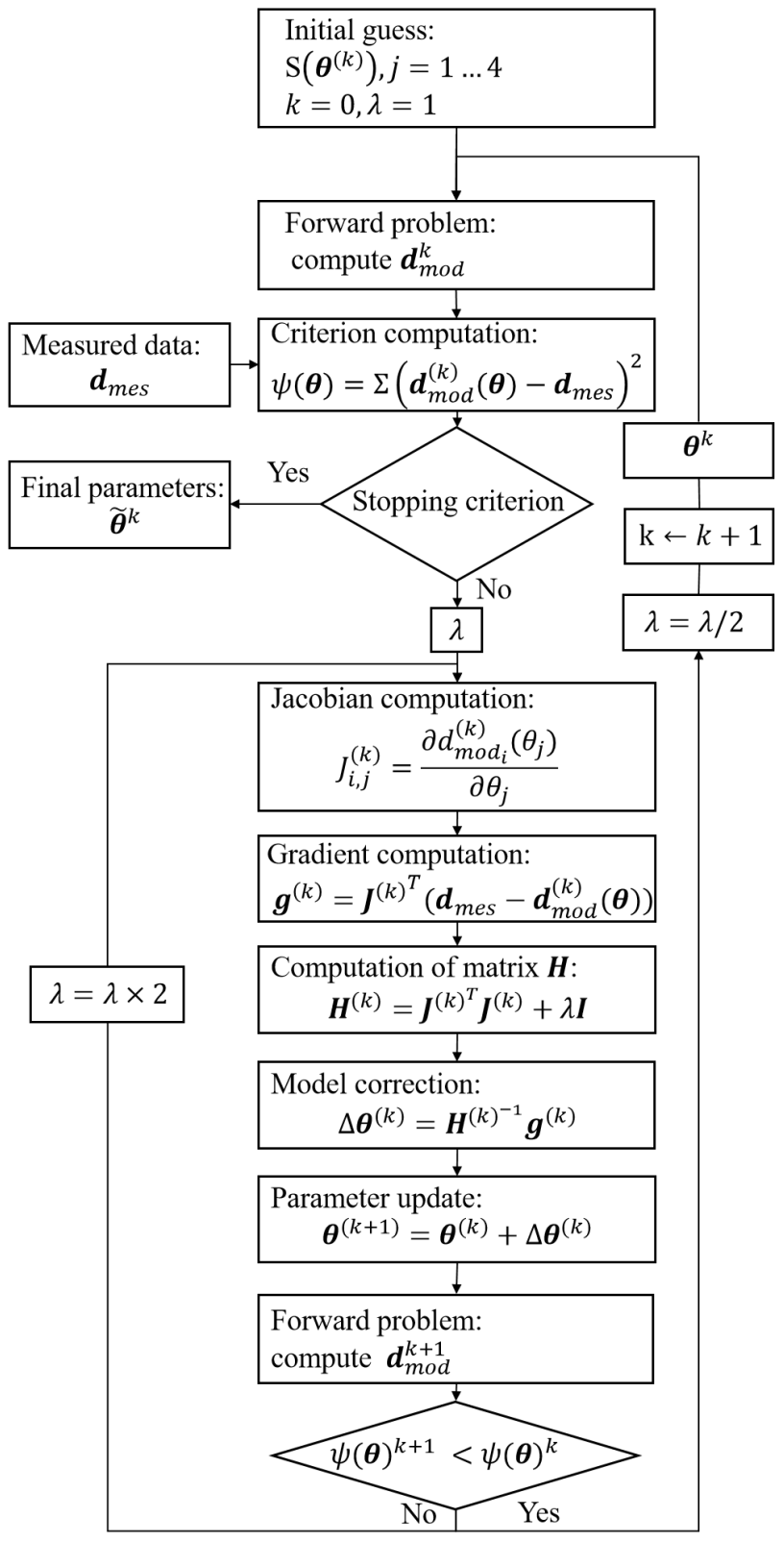

Figure 4: Levenberg-Marquardt inversion scheme, where $k$ is the iteration number, $\boldsymbol{J}$ is the Jacobian matrix and $\boldsymbol{g}$ is the gradient vector.

updated by computing the Jacobian matrix $\boldsymbol{J}$ (Eq. (10)), the gradient vector $\boldsymbol{g}$ (Eq. (11)) and the matrix $\boldsymbol{H}$ (Eq. (12)).

$J_{i, j}=\frac{\partial d_{\text {mod }_{i}}\left(\theta_{j}\right)}{\partial \theta_{j}}$

$\boldsymbol{g}=\boldsymbol{J}^{\mathrm{T}}\left(\mathbf{d}_{\text {mes }}-\mathbf{d}_{\text {mod }}(\boldsymbol{\theta})\right)$

$\boldsymbol{H}=\boldsymbol{J}^{\mathrm{T}} \boldsymbol{J}+\lambda \boldsymbol{I}$ 


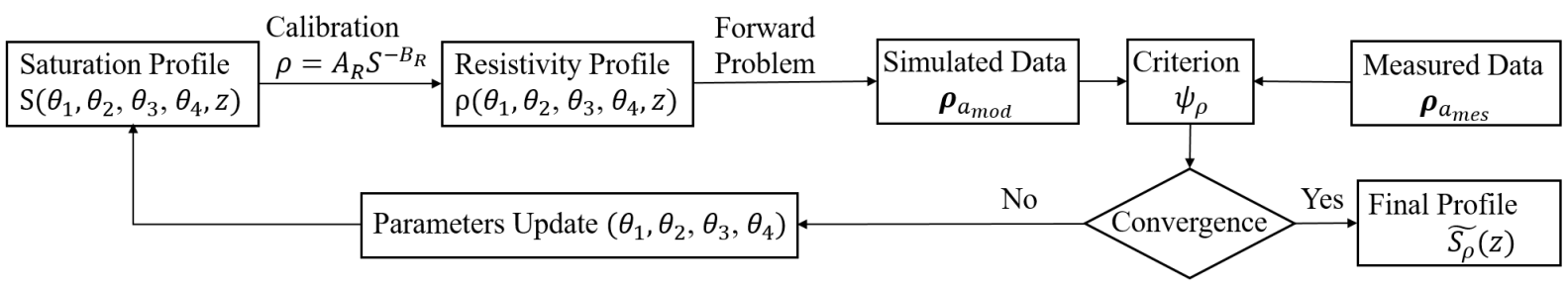

(a)

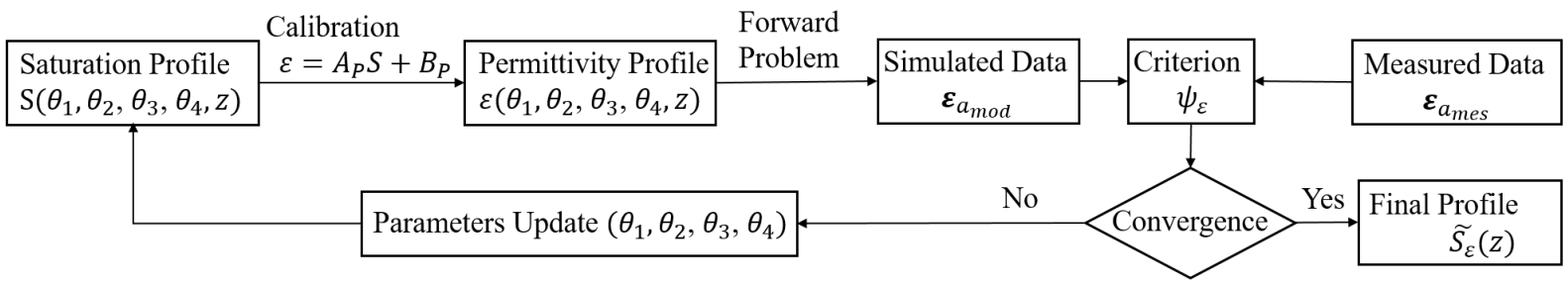

(b)

Figure 5: Inversion scheme of (a) apparent resistivity data into the profile of saturation degree versus depth (b) apparent permittivity data into the profile of saturation degree versus depth

Given that the data are modeled by the finite element method, we do not have access to the analytical expression of the relationship between parameters and data. Thus, the computation of the partial derivatives of the Jacobian matrix is approximated by the perturbation method [60]. Then, the procedure is repeated until fulfilling the stopping criteria. The inversion is stopped when the norm of the gradient vector $\boldsymbol{g}$ is less than a predefined threshold and set to $10^{-3}$ in this paper or when the number of iterations reaches a predefined limit.

\subsubsection{Separate inversion of resistivity and permittivity data}

The "separate" inversion consists of inverting only one type of electromagnetic measurements to estimate the saturation degree profile parameterized by the Weibull-type curve in Eq. (8). Considering that the calibration laws, established for a given concrete formulation in Eq. (6) and Eq. (7), can be used for the characterization of concrete at different water saturation degrees, the electromagnetic properties of the material are expressed using the saturation degree profile and these calibration laws.

The inversion method is similar for both types of measurements and is shown in Fig. 5. For the electrical method (Fig. 5(a)), an initial saturation degree profile is proposed. This profile is then converted into an electrical resistivity profile by applying the calibration law in Eq. (6) and the forward problem is solved to model the apparent resistivities. The minimization of the criterion $\psi_{\rho}$ given in Eq. (13) allows us to find the four optimal parameters of the saturation degree profile:

$\psi_{\rho}(\boldsymbol{\theta})=\sum_{i=1}^{n_{\rho}}\left(\rho_{i_{a_{m o d}}}(\boldsymbol{\theta})-\rho_{i_{a_{m e s}}}\right)^{2}$

The same applies for the inversion of the apparent permittivities where the goal is to minimize $\psi_{\varepsilon}$ in Eq. (14):

$\psi_{\varepsilon}(\boldsymbol{\theta})=\sum_{i=1}^{n_{\varepsilon}}\left(\varepsilon_{i_{a_{m o d}}}(\theta)-\varepsilon_{i_{a_{m e s}}}\right)^{2}$

A saturation degree profile is obtained for each separate inversion.

\subsubsection{Joint inversion}

\section{Measurement complementarity}

The main advantage of the joint inversion procedure is that both measurements being sensitive to saturation but differently, the information gathered may give a more accurate estimation of the saturation profile. More precisely, the 


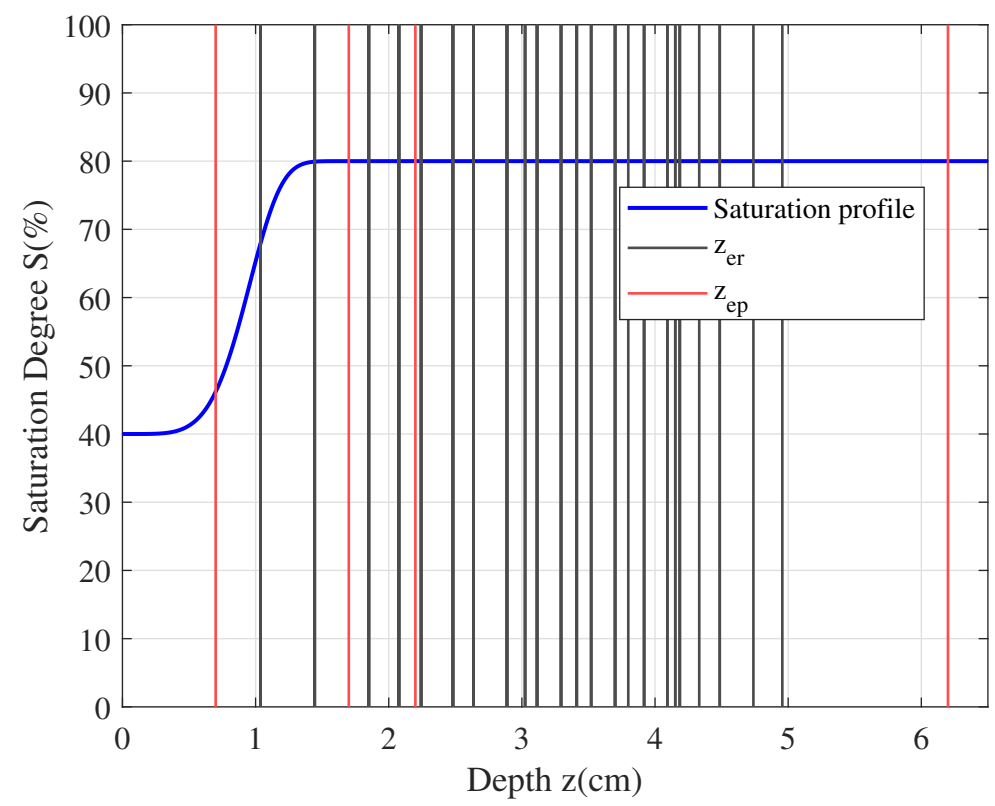

Figure 6: Estimated investigation depth for electrical (in black lines) [61] and capacitive measurement

$$
s \text { (in red lines) [26] for a typical saturation profile (blue line) }
$$

depth sensitivity of the two measurements is different. The devices used in this study (Section 2) were designed for the investigation of the concrete cover extending over the first 5 centimeters from the surface. Previous studies [61] and [26] have shown that knowing the spacing between electrodes, the investigation depth of these electrodes can be estimated. For the resistivity probe (Section 2.1.2), the investigation depth was estimated using analytical formulas given by [61]. The estimated thickness corresponds to $50 \%$ of the total sensitivity of the electrode configuration. Concerning the capacitive plates (Section 2.2.2), the investigation depth was estimated using numerical simulation with COMSOL Multiphysics [26]. The estimated thickness corresponds to $95 \%$ of the total sensitivity of the capacitive array. Figure 6 shows two sets of estimated investigation depths $z_{e}$. For resistivity measurements, the estimated investigation depths $z_{e r}$ are represented by black vertical lines and, for permittivity measurements, $z_{e p}$ are represented by red vertical lines. There are more black lines than red lines since the number of resistivity measurements is greater than the number of permittivity measurements. Figure 6 also shows a typical saturation profile represented by a blue line, indicating the measurement information distribution according to depth. We note that the range of targeted investigation depths is comparable for the two probes. These investigation depth values are also complementary and provide information on the entire thickness of the concrete cover. Therefore, combining electrical and capacitive measurements may lead to a better sampling of the concrete cover thickness, and therefore contribute to a better prediction of the saturation profile.

\section{Joint inversion algorithm}

Our joint inversion approach is described in Fig. 7. It is an iterative process which starts with an initial saturation profile given by the initial parameters $\boldsymbol{\theta}^{(0)}$. Then, it is converted into a resistivity profile using Eq. (6), and into a permittivity profile using Eq. (7). Afterwards, the simulated data that are the apparent resistivities and permittivities are computed (forward problems) and the data misfit criterion, $\psi$ given in Eq. (15), is computed.

$\psi(\boldsymbol{\theta})=\omega_{\rho} \sum_{i=1}^{n_{\rho}}\left(\rho_{i_{a_{\text {mod }}}}(\boldsymbol{\theta})-\rho_{i_{a_{m e s}}}\right)^{2}+\omega_{\varepsilon} \sum_{j=1}^{n_{\varepsilon}}\left(\varepsilon_{j_{a_{m o d}}}(\boldsymbol{\theta})-\varepsilon_{j_{a_{m e s}}}\right)^{2}$

where $\omega_{\rho_{i}}=\frac{1}{\sigma_{\rho_{i}}^{2}}, \omega_{\varepsilon_{j}}=\frac{1}{\sigma_{\varepsilon_{j}}^{2}}$ with $\sigma_{\rho_{i}}$ the standard deviation associated to each electrical measurement $i$ and $\sigma_{\varepsilon_{j}}$ the standard deviation associated to each capacitive measurement $j$.

$\psi$ is a linear combination of criteria $\psi_{\rho}$ (Eq. (13)) and $\psi_{\varepsilon}$ (Eq. (14)), weighted by $\omega_{\rho}$ and $\omega_{\varepsilon}$ which take into 


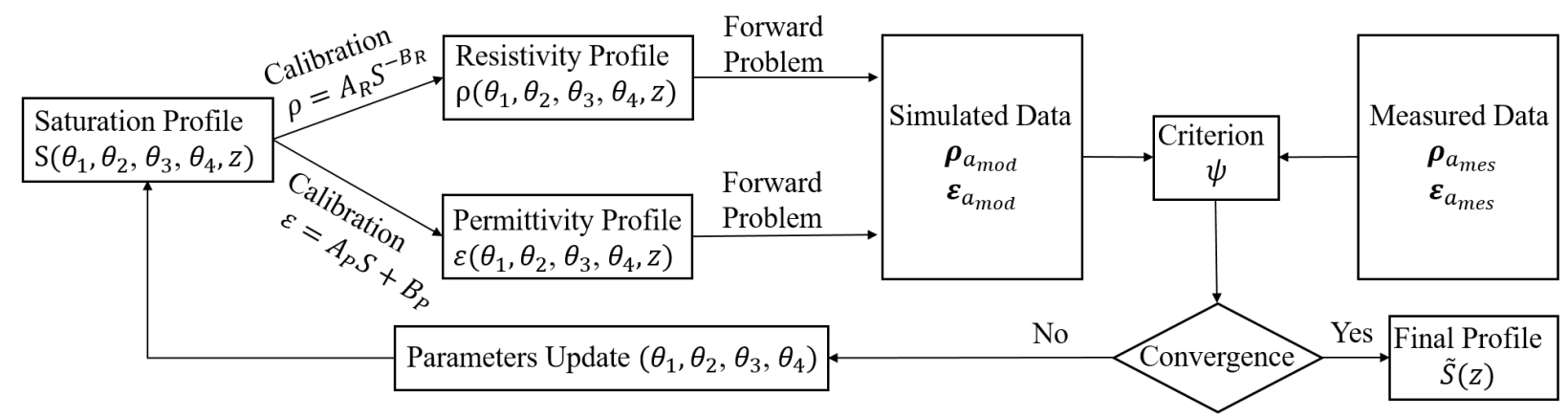

Figure 7: Joint inversion scheme to determine the concrete saturation degree depth profile from both resistivity and capacity measurements.

account the uncertainty of resistive and capacitive measurements respectively. Such weights correspond to the statistical assumption that the error/noise terms follow a Gaussian distribution with respective variance $\sigma_{\rho}^{2}$ and $\sigma_{\varepsilon}^{2}$. Then, the parameters are updated and a new iteration is run until $\psi$ is minimal according to the Levenberg-Marquardt algorithm described in Section 3.3.

\section{Numerical study}

A numerical study is presented in this section to validate the joint inversion method proposed.

\subsection{Study of sensitivity to the four parameters of the Weibull model}

First, the sensitivity of the data to the parameters of the model is studied. The design of experiments methodology was used to conduct this study [62]. It applies to many disciplines when the aim is to find the link between a quantity of interest and variables, to estimate the contribution of each of the parameters and their coupled effects to the quantity [63, 64, 65]. A multi-level experimental design was suggested from the Weibull parameters and their corresponding possible values, also known as levels. The parameter bounds are chosen to represent the physical domain of the possible and acceptable saturation degree profiles. The interval of each of the parameters is then discretized into several levels, to properly sample the variation space and to limit the computation time (about $1 \mathrm{~min} 30$ s for each "experiment"). An experimental design is then built including all the possible tests. A test consists of modeling the apparent data for a given saturation profile defined by a combination of the parameters and in computing the criterion for these data. To do so, it is necessary to have measured data. Therefore, 15 models were chosen, and the corresponding synthetic data are considered. This choice was made so that the study can represent the majority of possible saturation degree profiles.

For this case study, the experimental design is the following: the first two Weibull parameters $\theta_{1}$ and $\theta_{2}$ are given 7 possible levels and the two other parameters $\theta_{3}$ and $\theta_{4}$ are given 6 possible levels. Since $\theta_{1}$ and $\theta_{2}$ represent the two extreme saturation degrees, they are given values ranging from $30 \%$ to $95 \%$, which are typical saturation degree values for dry and humid concrete. $\theta_{3}$ represents the scale parameter, which locates the saturation edge. It is given values between 10 and $60 \mathrm{~mm}$, which are reasonable in the available depth of investigation range (Fig. 6). Finally, the shape parameter $\theta_{4}$, which impacts the slope of the saturation front, varies between 3 and 8 and is chosen depending on the form of the Weibull curve. The value $\theta_{4}=8$ corresponds to a steeper slope at the saturation front than the value $\theta_{4}=3$. For all possible parameter combinations, a total number of 1764 synthetic experiments is carried out. For each one of them, $\rho_{a_{\text {mod }}}$ and $\varepsilon_{a_{\text {mod }}}$ are modeled (Section 3.2) and the criterion Eq. (15) is computed. Once all experiments are simulated, the analysis of variance (ANOVA) test is performed to analyze the influence of each parameter. The results are shown in Fig. 8 representing the contribution of each parameter to the explanation of the total variance. Parameters $\theta_{1}, \theta_{2}$ and $\theta_{3}$ have the most important contribution to the total variance, as well as their coupled effect $\left(\theta_{1} \theta_{2}, \theta_{1} \theta_{3}\right.$ and $\theta_{2} \theta_{3}$ ), whereas parameter $\theta_{4}$ has a negligible contribution. This leads to conclude that a variation for $\theta_{4}$ does not lead to an important change of the criterion. Hence, it should be more difficult to estimate parameter $\theta_{4}$ than any other parameter. 


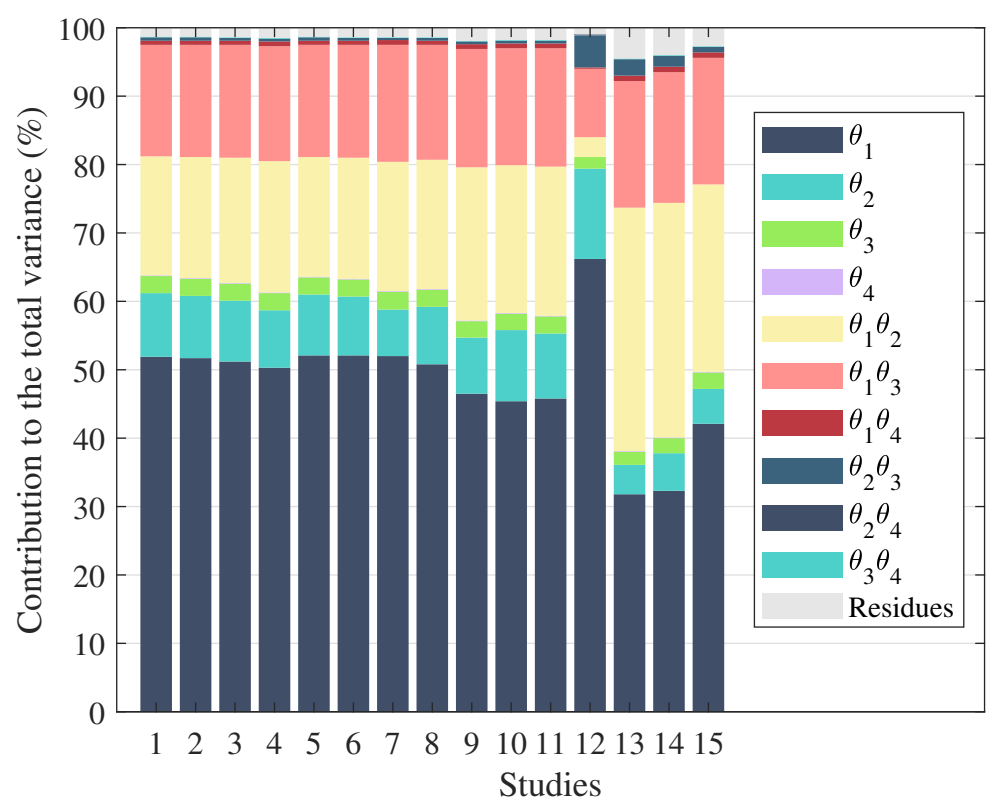

Figure 8: Contribution of the parameters $\theta_{i}$ and of the products $\theta_{i} \theta_{j}, i, j=1, \ldots, 4$, to the total variance of criterion $\psi$.

\subsection{Inversion of synthetic data}

The joint inversion method is now tested on synthetic data. The forward model is computed using the numerical slab model and electrode simulation presented in Section 3.2. The numerical experiments are performed as follows: a saturation profile is generated according to Eq. (8) with parameters given in Table 1, column 2. In all simulations, the initial saturation profile in the optimization procedure is chosen such that a deterministic relative difference compared to the true profile is equal to $10 \%$ (Table 1 , column 3 ).

In the first experiment, we consider noise-free data. The inversion procedure is run successively by considering only apparent resistivity data, only apparent permittivity data, and finally both data sets. The results are shown in Fig. 9. Profiles corresponding to the successive iterations of the Levenberg-Marquardt procedure are represented by the green curves. It is clear that the three inversion methods correctly converge toward the true profile. Also, in the inversion of apparent permittivity data (Fig. 9(b)), although each iteration improves the fit of the data, the algorithm tends to estimate intermediate profiles that move away from the true profile. This variability disappears with the joint inversion (Fig. 9(c)) due to the addition of resistivity data, which induces a faster convergence (15 iterations for the joint inversion compared to 37 for the permittivity inversion alone). Comparing the results of the resistivity inversion and the joint inversion, both inversion approaches have similar performances; the resistivity inversion approach requires 11 iterations and the joint inversion approach requires 15 iterations.

The second inversion test aims to study the influence of noise. Referring to previous noise assessment studies for capacitive and resistive measurements on concrete specimen [7], two noise levels for each type of measurement are considered and are represented by their coefficient of variation defined as the ratio of the standard deviation to the mean of the measurements. For resistive measurements, the two noise levels correspond to the variation coefficients $C V_{r}=4 \%$ and $12 \%$ (representative of low and high noise level). Note that, in practice, measurements in dry concrete can be much noisier. For capacitive measurements, $C V_{p}=2 \%$ and $6 \%$. Then, the saturation profile defined by the same parameters as in the first experiment is considered. An initial model deviated by $10 \%$ from the true model is chosen, as in the case of the first experiment. Gaussian noise is then added to the data for each noise level, and the procedure is repeated 10 times. Separate and joint inversions are performed for each random realization. Then we focus on the average of the 10 estimated models, on the standard deviation of each parameter of the "estimated profile", on the number of iterations and the average relative error between the parameters of the estimated model and the parameters of the true model. Results are given in Table 1. For the low noise level $\left(C V_{r}=4 \%\right.$ and $\left.C V_{p}=2 \%\right)$, the relative error of the model obtained by the joint inversion procedure as well as the variabilities of the parameters of the estimated model are the lowest. For the high noise level $\left(C V_{r}=12 \%\right.$ and $\left.C V_{p}=6 \%\right)$, the model estimated by the joint inversion is slightly 


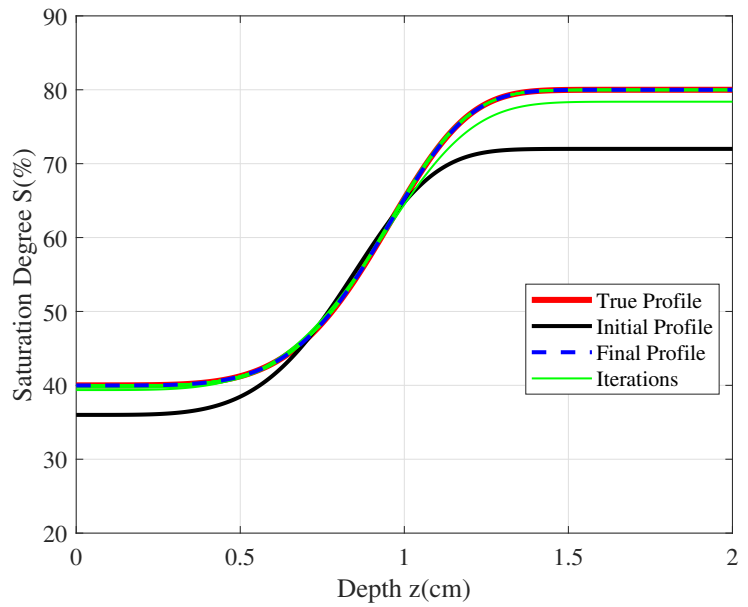

(a)

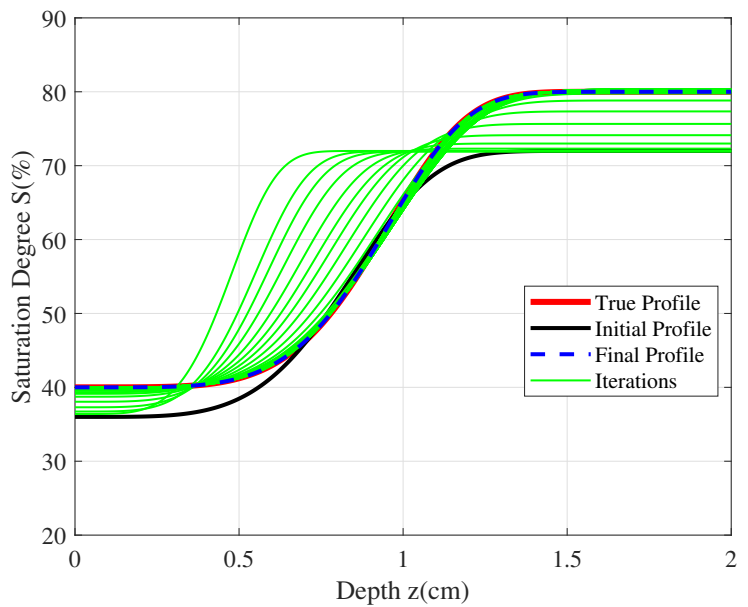

(b)

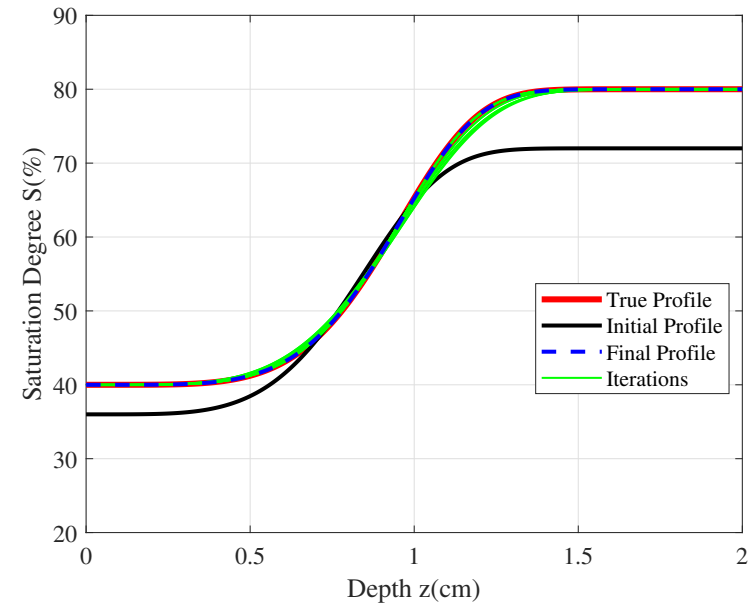

(c)

Figure 9: (a) Inversion procedure using noise-free apparent resistivity data alone (b) Inversion of noise-free apparent permittivity data alone and (c) Joint inversion of noise-free apparent resistivity and permittivity data.

more distant from the true model than the model estimated by the resistivity inversion. This is mainly due to the fourth parameter $\theta_{4}$ which did not converge well to its true value. For both permittivity inversions (column 6), the relative errors between the estimated and the true model parameters are the highest. This can be explained by the very small number of capacity data compared to the number of resistivity data (see Section 2), such that the fit between the data and the model is poorly constrained due to insufficient information gathered from the capacitive method. Furthermore the maximum number of iterations (set to 50) is reached. Comparing the results for the different noise levels, we notice first that the joint inversion procedure requires fewer iterations than the resistivity and the permittivity inversion procedures. Furthermore, the higher the noise level, the more the estimated final model differs from the true model and it is especially the fourth parameter that does not seem to converge well towards the true value.

\section{Experimental study}

\subsection{Experimental setup}

In this section, our joint inversion procedure is applied to experimental laboratory data. The drying of an initially saturated concrete slab is considered. The concrete slab, designed for laboratory measurements, is kept in a controlled temperature during the drying process $\left(\mathrm{T}=20^{\circ} \mathrm{C} \pm 0.8^{\circ} \mathrm{C}\right)$. The relative humidity in the room varies between $42 \%$ and $60 \%$. The electric and capacitive measurements are made on a slab of dimensions $500 \times 250 \times 120 \mathrm{~mm}^{3}$. The slab 
Joint inversion of electromagnetic measurements for the determination of water saturation profiles in concrete structures

Table 1

Results of the separate and joint inversions of the apparent resistivity and permittivity data considering low and high noise levels.

\begin{tabular}{lllllll}
\hline Parameters & $\begin{array}{l}\text { True } \\
\text { Profile }\end{array}$ & $\begin{array}{l}\text { Initial } \\
\text { Profile }\end{array}$ & $\begin{array}{l}\text { Noise } \\
\text { Conditions }\end{array}$ & $\begin{array}{l}\text { Resistivity } \\
\text { Inversion }\end{array}$ & $\begin{array}{l}\text { Permittivity } \\
\text { Inversion }\end{array}$ & $\begin{array}{l}\text { Joint } \\
\text { Inversion }\end{array}$ \\
\hline$\theta_{1}$ & 40 & 36 & $C V_{r}=4 \%$ & $39.27 \pm 1.76$ & $40.31 \pm 6.5$ & $39.91 \pm 0.70$ \\
$\theta_{2}$ & 80 & 72 & $C V_{p}=2 \%$ & $80.06 \pm 0.26$ & $95.00 \pm 18.25$ & $\begin{array}{l}79.67 \pm 0.69 \\
\theta_{3}\end{array}$ \\
$\theta_{4}$ & 5.01 & 0.011 & & $0.0101 \pm 0.0003$ & $0.0119 \pm 0.012$ & $0.0100 \pm 0.0002$ \\
& 5 & 4.5 & & $4.996 \pm 0.0025$ & $10.85 \pm 10.1$ & $4.9811 \pm 0.75$ \\
& & & Nb of iterations & 10 & 50 & 6 \\
$\theta_{1}$ & 40 & 36 & $C V_{r}=12 \%$ & $39.65 \pm 4.11$ & $39.97 \pm 5.17$ & $40.10 \pm 1.65$ \\
$\theta_{2}$ & 80 & 72 & $C V_{p}=6 \%$ & $79.84 \pm 0.52$ & $82.32 \pm 35.63$ & $80.28 \pm 0.56$ \\
$\theta_{3}$ & 0.01 & 0.011 & & $0.0102 \pm 0.0009$ & $0.0074 \pm 0.0147$ & $0.0100 \pm 0.0010$ \\
$\theta_{4}$ & 5 & 4.5 & & $4.4991 \pm 0.001$ & $58.115 \pm 46.8$ & $5.6953 \pm 2.23$ \\
& & & Nb of iterations & 12 & 50 & 10 \\
& & & Relative error & $3.28 \%$ & $272 \%$ & $3.70 \%$ \\
\hline
\end{tabular}

Table 2

Composition of concrete for the experimental campaign

\begin{tabular}{llll}
\hline Component & & Source & Quantity $\left(\mathrm{kg}, \mathrm{m}^{3}\right)$ \\
\hline Aggregate & $4 / 12$ & Quarry of Boulonnais & 984 \\
\hline Sand & $0 / 4$ & Quarry of Boulonnais & 890 \\
\hline Cement & CEMI 52,5N & Lafarge, Val d'Azergues & 350 \\
\hline Admixtures & AD & MasterGlenium SKY 537 & 0 \\
\hline Water & & & 206 \\
\hline
\end{tabular}

is made of concrete mixed with CEMI Portland cement. The water-to-cement ratio is 0.59. Table 2 summarizes the composition of concrete. The compressive strength at 28 days is $42.6 \mathrm{MPa}$, with a standard deviation of $1.3 \mathrm{MPa}$, and the porosity at 28 days is $15.3 \%$, with a standard deviation of $0.3 \%$. To ensure a homogeneous initial saturated state, the slab was completely immersed in water for more than one year. Then, at time $T_{0}$, the slab was removed from water and kept at a controlled temperature of $20^{\circ} \mathrm{C} \pm 0.8^{\circ} \mathrm{C}$ to dry. The four small faces were protected with an aluminum film so that the water evacuation occurs from the two remaining faces. The measurement campaign took place at nine times: $T_{0}, T_{1}=T_{0}+17$ days, $T_{2}=T_{0}+21$ days, $T_{3}=T_{0}+29$ days, $T_{4}=T_{0}+42$ days, $T_{5}=T_{0}+56$ days, $T_{6}=T_{0}+69$ days $T_{7}=T_{0}+84$ days and $T_{8}=T_{0}+104$ days. At each time, the measurements were performed on the two drying faces.

Resistivity measurements were made using a SYSCAL Pro resistivity meter (IRIS Instruments) that allows to significantly mitigate polarization issues by transmitting a low-frequency $(2 \mathrm{~Hz})$ alternating square-shaped current and by applying some signal preprocessing to remove artifacts due to remaining polarization. Apparent resistivities were measured with the 14-electrode resistivity probe described in Section 2. In Fig. 10, the electrodes are represented by 14 blue squares on the block surface and aligned on the diagonal of the rectangle. Six measurements were made on each face: two measurements with three different orientations (Fig. 10(a)). To obtain the apparent resistivity, the measured transfer resistances (V/I) were then multiplied by the corresponding geometric factors $G_{R}$, modeled using the finite element method (see Section 2.1.2). The mean value and standard deviation of the six measurements were computed for each drying face of the slab. to represent the evolution of the apparent resistivity as a function of time and to perceive the establishment of the electrical resistivity profile due to the water content gradient in concrete, the apparent resistivity values are represented as a function of the investigation depth in Fig. 11(a). The measurements on both front and rear drying faces are presented by solid and dotted lines respectively on the same side in Fig. 11(a). At time $T_{0}$, the apparent resistivity is the lowest and it is almost constant as a function of the investigation depth. This corresponds to the saturated state of concrete where the measured apparent resistivities are approximately equal to 35 


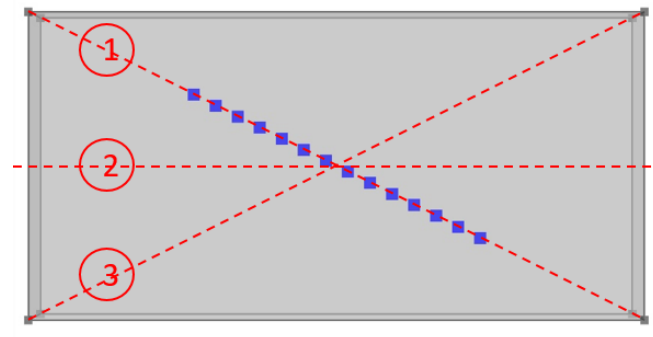

(a)

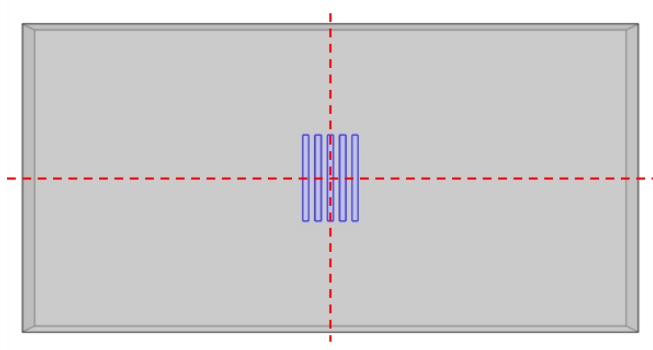

(b)

Figure 10: Electrodes and measurement position for (a) resistivity and (b) permittivity measurement

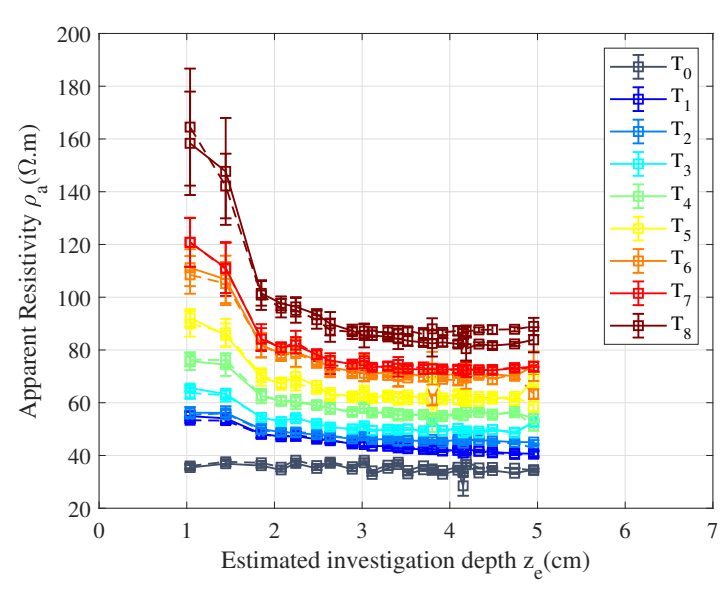

(a)

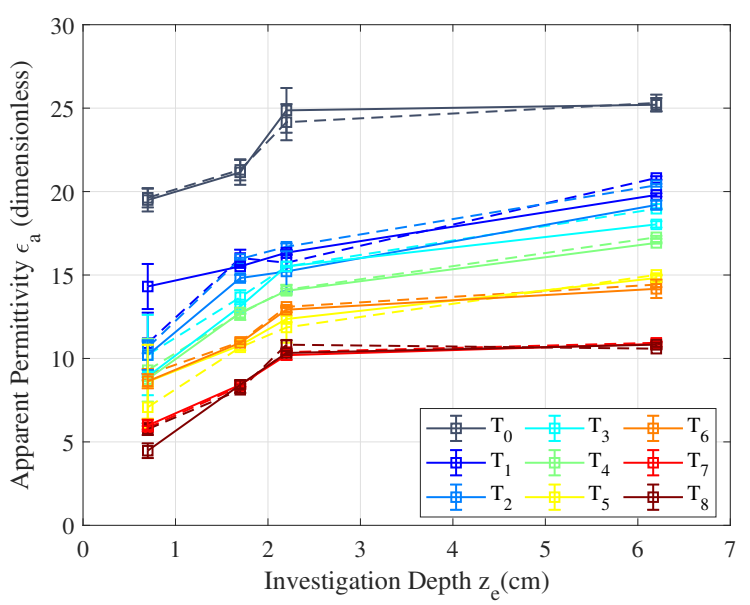

(b)

Figure 11: Measurement results for both front and rear drying faces (represented by solid and dashed lines respectively) at 9 different test times (a) apparent resistivity measurements (b) apparent permittivity measurements

S.m. As could be expected, the apparent resistivity increases with time due to concrete drying. In addition, at each time, the apparent resistivity decreases with the investigation depth, reflecting the fact that the concrete water saturation degree increases with depth.

Permittivity measurements were made using the capacitor and probes described in Section 2.2.2. In Fig. 10(b), the electrodes are represented by blue plates of negligible thickness and placed on the center block surface. At each time, about ten measurements were made with each probe on each drying face. First, for each probe, the system resonant frequency in the air is measured. Then, the resonant frequency in concrete is measured to obtain the apparent permittivity using Eq. (4). The mean value and the standard deviation of all measurements were computed for each probe and each drying face of the slab. In Fig. 11(b), the apparent permittivities obtained on the slab are shown for each time instance. At time $T_{0}$, the apparent permittivity is the highest (around 25) and it is almost constant as a function of the investigation depth. This corresponds to the saturated state of concrete. Similar observations can be made than for resistivity measurements: due to the drying process, the apparent permittivity naturally decreases with time and increases with depth.

Finally, Fig. 11 shows that apparent resistivity and permittivity measurements are very close for the two drying faces at all test times which shows the symmetrical drying of concrete and the good measurement quality. 


\subsection{Inversion of experimental data}

Fig. 12 shows the estimated saturation profiles obtained by the individual and joint inversion procedures described in Section 3.3, obtained independently for each time instance and for each face of the slab.

Fig. 12(a) shows the estimated saturation profiles using only apparent resistivity data. For both front and rear drying faces, the profile decreases with time and increases with depth, following the resistivity measurements. For solid line profiles, it decreases from $88 \%$ (at $T_{1}$ ) to $53 \%$ (at $T_{8}$ ) on the surface, and from $100 \%$ to $79 \%$ in depth. For dashed profiles, it decreases from $85 \%$ to $56 \%$ on the surface and from $98 \%$ to $77 \%$ in depth between $T_{1}$ and $T_{8}$. Also, for both faces, we can observe the progress of the drying front with time, reaching $1.4 \mathrm{~cm}$ for the last instance. Note that the estimated profiles obtained on the two faces are very similar at all measurement times, confirming the reproducibility of the apparent resistivity measurements observed in Fig. 10 and the validity of the inversion procedure.

Fig. 12(b) shows the estimated saturation profiles using only apparent permittivity data. For both faces, the profile also decreases with time and increases with depth. For solid line profiles, it decreases from $63 \%$ to $11 \%$ on the surface and from $100 \%$ to $48 \%$ in depth between $T_{1}$ and $T_{8}$. For dashed profiles, it decreases from $64 \%$ to $12 \%$ on the surface and from $100 \%$ to $56 \%$ in depth between $T_{1}$ and $T_{8}$. For both drying faces, we can also observe slight progress of the drying front with time, reaching $0.2 \mathrm{~cm}$ for the last instance.

Fig. 12(c) finally shows the estimated saturation profiles using both apparent resistivity and permittivity data. Once again, all profiles naturally decrease with time. For solid line profiles, it decreases from $96 \%$ to $33 \%$ on the surface and from $100 \%$ to $60 \%$ in depth between $T_{1}$ and $T_{8}$. For dashed profiles, it decreases from $91 \%$ to $40 \%$ on the surface and from $90 \%$ to $60 \%$ in depth between $T_{1}$ and $T_{8}$. Using both data sets, the progress of the drying front is now estimated at $1.9 \mathrm{~cm}$ for the last instance. Here also, the profiles estimated for each face of the slab are very similar at all measurement times.

The results analysis clearly shows the effect of water evaporation over time. However, for each time instance, the estimated profiles are not the same using the three types of data inversion. In particular, greater values of surface saturation degrees are obtained using resistivity data (Fig. 12(a)) than with permittivity data (Fig. 12(b)). This difference may be due to the calibration curves used for the saturation degree (Eq. (6) and Eq. (7)) showing the difference between the sensitivity of the two types of measurements to the saturation degree. As could be expected, the estimated profiles using both data sets (Fig. 12(c)) are in between.

\section{Discussion}

Measurements using the gammadensimetry method [66] were performed on a concrete specimen to obtain some additional "ground truth" measurements of the saturation profile at different depths. Measurements were performed on a $\phi 110 \times 300 \mathrm{~mm}$ cylindrical specimen that has the same concrete composition as the one studied in this paper. A unidirectional drying at a controlled temperature of $20^{\circ} \mathrm{C}$ was also followed for the gammadensimetry concrete specimen. The principle of the gammadensimetry method is based on the absorption of gamma photons emitted by a radioactive source when they pass through a concrete sample. The received gamma photons are then converted into electrical pulses and are counted for a given time. The work in [66] shows a relationship between the number of photons counted during a time interval and the saturation degree of a certain volume of a concrete slice. The cylindrical specimen is rotating around its axis. Therefore, the virtual concrete slice is defined by the photon beam diameter (height) and the specimen diameter. The counting is repeated for several virtual slices to obtain the saturation degree profile as a function of depth. The uncertainty in measuring the degree of saturation by this method is of the order of $3 \%$. At $T_{4}=T_{0}+42$ days, measurements using both gammadensimetry and non-destructive techniques were performed. Fig. 13 shows the inverted profiles obtained by the three types of data inversion compared to the gammadensimetry measurements at this time. We considered the NRMSE (Normalized Root Mean Square Error) between the gammadensimetry curve and the ones obtained by inversion defined by:

$N R M S E=\frac{\sqrt{\frac{\sum_{i=1}^{N}\left(S_{g}-S_{i}\right)^{2}}{N}}}{S_{\text {max }}-S_{\text {min }}}$

where $S_{g}(\%)$ is the saturation degree of the gammadensimetry curve, $S_{i}(\%)$ the saturation degree of the curve estimated by data inversion, $S_{\min }(\%)$ and $S_{\max }(\%)$ respectively the minimum and maximum value of the saturation degree. NRMSE values were high (70.9\%) for the inverted apparent permittivity, significantly lower (19.4\%) for the inverted 


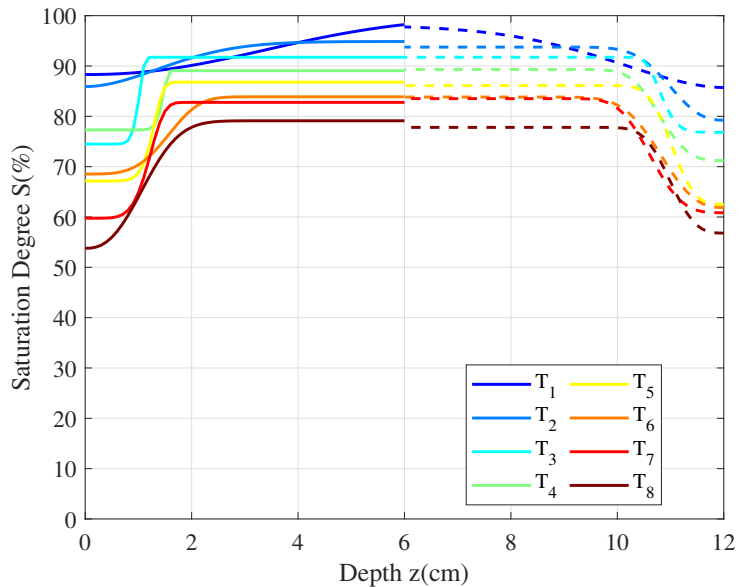

(a)

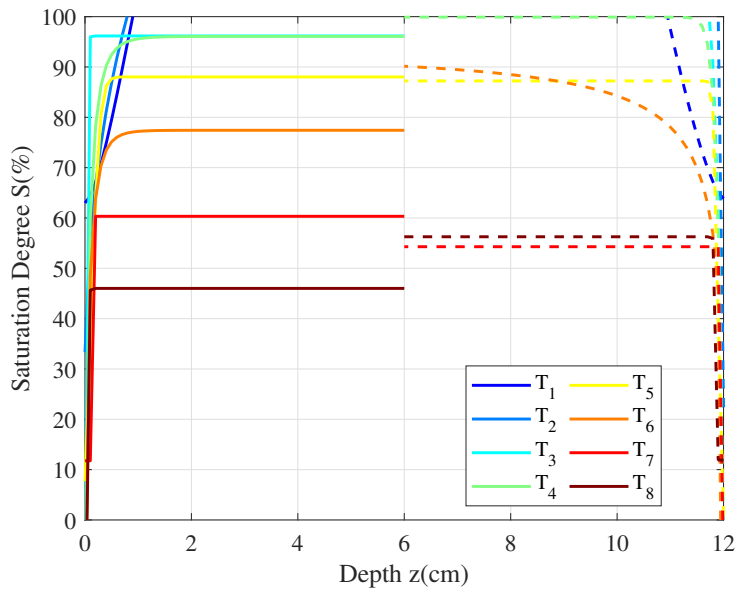

(b)

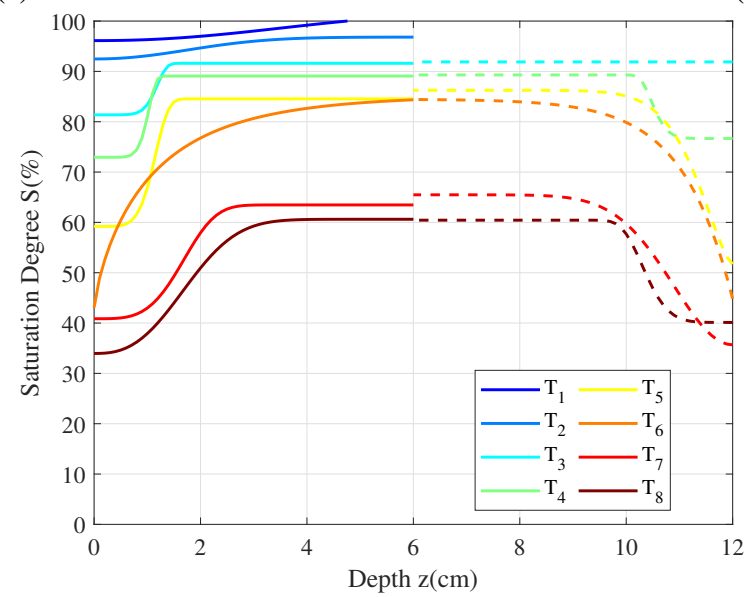

(c)

Figure 12: Inversion results for both front and rear drying faces in plain and dotted lines respectively using (a) apparent resistivity data alone (b) apparent permittivity data alone and (c) both apparent resistivity and permittivity data.

apparent resistivity and the lowest (16.9\%) for the curve obtained with the joint inversion of both data sets, showing that the saturation profiles estimated by resistivity and joint inversion at time $T_{4}$ are physically plausible.

The estimated profiles using the joint inversion approach are consistent with the evolution of the state of the concrete during the drying phenomenon. Comparing these profiles with those estimated by separate inversion, several improvements are observed. The profiles estimated by joint inversion are, on the one hand, characterized by a more advanced drying front than that of the profiles estimated by inversion of the apparent resistivities (Fig. 12 and Section 5.2) and, on the other hand, are more realistic and consistent than those estimated by inversion of the apparent permittivities. These results validate, on experimental data, the benefits of exploiting the complementarity of the two types of measurements.

Furthermore, Table 3 shows the average iteration number and computation time for the three types of data inversion. The slowest approach is the one using permittivity data alone, where each inversion requires about 135 min and 50 iterations to reach convergence. This can be explained, as for the synthetic data inversion (Section 4.2), by the very small number of capacity data compared to the number of resistivity data, such that the information gathered from the capacitive method is insufficient. The resistivity inversion and the joint inversion procedures have comparable computation times, with slightly higher times for the joint inversion approach. This extra time is due to the extra computation of the capacitive forward model (requiring approximately $1 \mathrm{~min}$ ) at each iteration of the joint inversion procedure. Nonetheless, this is mostly compensated by a strong decrease in the number of iterations required by the 
Joint inversion of electromagnetic measurements for the determination of water saturation profiles in concrete structures

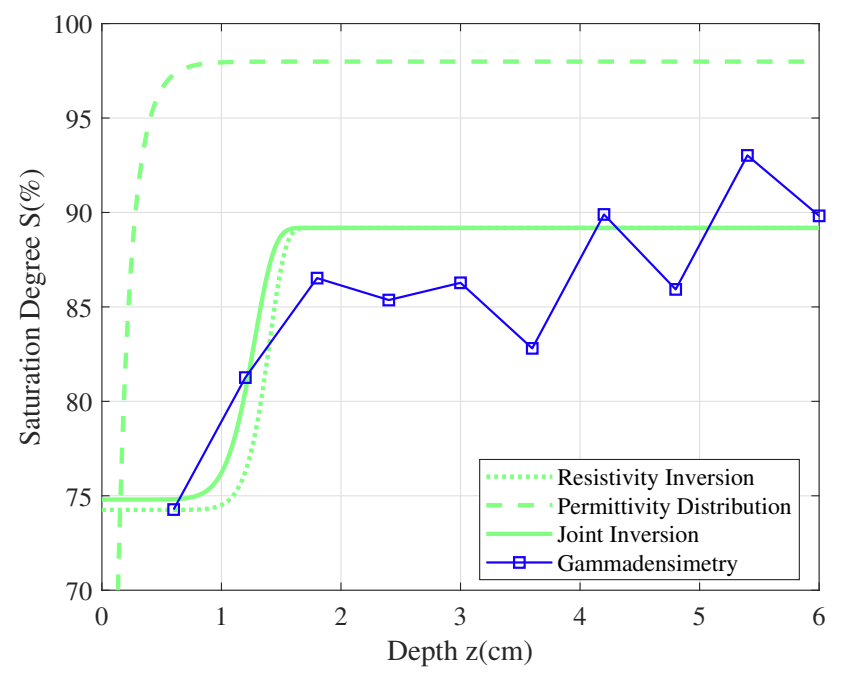

Figure 13: Comparison of inverted profiles obtained with different inversion approaches to gammadensimetry measurement at $T_{4}=T_{0}+42$ days

\section{Table 3}

Computation time and iteration number for experimental data inversion using different inversion procedures (average \pm standard deviation).

\begin{tabular}{lll}
\hline Data Used for Inversion & Computation Time $(\mathrm{min})$ & Iteration Number \\
\hline Apparent resistivity & $27 \pm 9$ & $15 \pm 9$ \\
\hline Apparent permittivity & $135 \pm 18$ & $50 \pm 0.25$ \\
\hline Apparent resistivity and permittivity & $35 \pm 6$ & $5 \pm 3$ \\
\hline
\end{tabular}

Levenberg-Marquardt algorithm. The joint inversion approach requires three times fewer iterations to converge than the individual resistivity inversion.

\section{Conclusion}

This paper presents a new inversion approach for non-destructive resistivity and permittivity data in concrete structures based on the combination of these data. Indeed, using calibration curves connecting saturation degree and electrical resistivity on the one hand, and saturation degree and dielectric permittivity on the other hand, the combination of the two types of non-destructive data is performed to predict concrete saturation degree distribution which is an important durability indicator. The joint inversion approach proposed is found to be effective to combine non-destructive electric and capacitive measurements to determine water saturation profiles in concrete structures.

Our numerical study showed that parameters $\theta_{1}$ to $\theta_{3}$ have a non-negligible contribution to the total variance, as well as their coupled effect, whereas parameter $\theta_{4}$ has a negligible contribution to the total variance of the criterion. Therefore, it is more difficult to estimate parameter $\theta_{4}$ than any other parameter. Afterwards, the three data inversion types (using resistivity data alone, then permittivity data alone, and finally joint inversion) tested on synthetic noise-free data correctly converge toward the true profile and that the joint inversion is the fastest to converge. When performing the joint inversion on synthetic noisy data, both the iteration number and the relative error are the lowest compared to inversion using resistivity or permittivity data alone. When a high noise level is considered, the estimation of parameter $\theta_{4}$ becomes difficult and this could be due to its negligible contribution to the data sensitivity.

Finally, when applying the three data inversion types to real experimental measurements performed during the drying of initially saturated concrete, the effect of water evaporation with time is observed. Moreover, the similarity of reconstructed saturation profiles between the two drying faces shows the quasi-symmetry of the drying phenomenon. In addition, comparing profiles estimated by joint inversion to gammadensimetry measurements performed at the same 
time, we noticed that the inverted profile is physically plausible since it is close to the measured one. At each time, the inverted saturation profile obtained from joint inversion is intermediate to the ones obtained using resistivity data alone and permittivity data alone. Then, it is found that the apparent resistivity inversion and joint inversion have the optimal computation time and that joint inversion requires three times fewer iterations than resistivity inversion to reach convergence.

Some suggestions for further research can be made to improve the proposed joint inversion approach. Since calibration curves are essential to connect two types of measurements to one variable of interest, it would be interesting to study the possibility of optimizing the calibration curve constants as part of the saturation model. This study can be justified by the fact that calibration constants are measured using experimental methods on a concrete specimen and may vary due to concrete aging. Furthermore, we aim to develop other capacitive electrode sets in future studies, so the information gathered from apparent permittivity measurements can be increased. In addition, the influence of the reinforcements on the apparent measurements by non-destructive methods, especially by the electrical method, is not negligible. However, this effect can be taken into account in the forward modeling of the apparent measurements [4]. Therefore, interesting further developments may extend the application of joint inversion to measurements carried out in the presence of reinforcements. Finally, the joint inversion approach presented herein can lead to study more complex phenomena such as wet/dry cycles, where a more flexible model parametrization than the Weibull one should be considered.

\section{Acknowledgements}

The authors would like to thank Région Pays de la Loire and Université Gustave Eiffel for their financial support, Andra for financing the experimental research project, Joanna Badr (Université Gustave Eiffel), Jean-Paul Balayssac (Université de Toulouse, LMDC laboratory) and MAST-LAMES laboratory of Université Gustave Eiffel for their contribution to the experimental campaign.

\section{References}

[1] M Saleem, M Shameemt, SE Hussain, and M Maslehuddintf. Effect of moisture, chloride and sulphate contamination on the electrical resistivity of Portland cement concrete. Construction and Building Materials, 10(3):209-214, 1996.

[2] JP Balayssac and V Garnier. Non-destructive Testing and Evaluation of Civil Engineering Structures. Press Ltd \& Elsevier Press Ltd., London, 2017.

[3] JF Lataste, G Villain, and JP Balayssac. Chapter 4. Electrical Methods. In Non-destructive Testing and Evaluation of Civil Engineering Structures. ISTE Press Ltd \& Elsevier Press Ltd., London, 2017.

[4] MA Alhajj, S Palma-Lopes, and G Villain. Accounting for steel rebar effect on resistivity profiles in view of reinforced concrete structure survey. Construction and Building Materials, 223:898-909, 2019.

[5] E Hammond and TD Robson. Comparison of Electrical Properties of Various Cements and Concretes. The Engineer, 199(5156):78-80, 1955.

[6] WJ McCarter, MC Forde, and HW Whittington. Resistivity Characteristics of Concrete, Proceedings. Institute of Civil engineers, 71:107-117, 1981.

[7] R Du Plooy, S Lopes, G Villain, and X Dérobert. Development of a multi-ring resistivity cell and multi-electrode resistivity probe for investigation of cover concrete condition. NDT \& E International, 54:27-36, 2013.

[8] WJ McCarter and S Garvin. Dependence of electrical impedance of cement-based materials on their moisture condition. Journal of Physics D: Applied Physics, 22(11):1773-1776, 1989.

[9] R Spragg, J Castro, T Nantung, M Paredes, and J Weiss. Variability Analysis of the Bulk Resistivity Measured Using Concrete Cylinders. Advances in Civil Engineering Materials, 1(1):1-17, 2012.

[10] DJ Smyl, M Hallaji, A Seppänen, and M Pour-Ghaz. Quantitative electrical imaging of three-dimensional moisture flow in cement-based materials. International Journal of Heat and Mass Transfer, 2016.

[11] DJ Smyl. Relating unsaturated electrical and hydraulic conductivity of cement-based materials. Australian Journal of Civil Engineering, 16(2):129-142, jul 2018.

[12] MH Loke and RD Barker. Rapid least-squares inversion of apparent resistivity pseudosections by a quasi-Newton method. Geophysical Prospecting, 44(1):131-152, 1996.

[13] M Chouteau and S Beaulieu. An investigation on application of the electrical resistivity tomography method to concrete structures. In The 2nd Annual Conference on the Application of Geophysical and NDT Methodologies to Transportation, 2002.

[14] W Daily, A Ramirez, A Binley, and D LeBrecque. Electrical resistance tomography. The Leading Edge, 23(5):438-442, 2004.

[15] MN Soutsos, JH Bungey, SG Millard, MR Shaw, and A Patterson. Dielectric properties of concrete and their influence on radar testing. NDT \& E International, 34(6):419-425, 2001.

[16] S Laurens, JP Balayssac, J Rhazi, and G Arliguie. Influence of concrete moisture upon radar waveform. Materials and Structures, 248:198-203, 2002.

[17] G Klysz and JP Balayssac. Determination of volumetric water content of concrete using ground-penetrating radar. Cement and Concrete Research, 8:1164-1171, 2007. 
[18] W Morris, EI Moreno, and AA Sagüés. Practical evaluation of resistivity of concrete in test cylinders using a Wenner array probe. Cement and Concrete Research, 1996.

[19] B Bing Zhou and T Dahlin. Properties and effects of measurement errors on 2D resistivity imaging surveying. Near Surface Geophysics, pages 105-117, 2003.

[20] T Dahlin and B Zhou. A numerical comparison of 2D resistivity imaging with 10 electrode arrays. Geophysical prospecting, 52:379-398, 2004.

[21] X Derobert. Presentation of common non destructive techniques, Capacitive technique. In D Breysse, editor, Non-Destructive Assessment of Concrete Structures: Reliability and Limits of Single and Combined Techniques, chapter 2, pages 71-77. Toulouse, 2012.

[22] P Gu and J Beaudoin. Dielectric behavior of hardened cement paste systems. Journal of materials science letters, $15,1996$.

[23] S Laurens, JP Balayssac, J Rhazi, G Klysz, and G Arliguie. Non-destructive evaluation of concrete moisture by GPR: Experimental study and direct modeling. Materials and Structures, 38(9):827-832, 2005.

[24] R Du Plooy, G Villain, S Palma Lopes, A Ihamouten, X Dérobert, and B Thauvin. Electromagnetic non-destructive evaluation techniques for the monitoring of water and chloride ingress into concrete: a comparative study. Materials and Structures, 48(1):369-386, jan 2015.

[25] G Villain, V Garnier, M Sbartai, X Dérobert, and JP Balayssac. Development of a calibration methodology to improve the on-site non-destructive evaluation of concrete durability indicators. Materials and Structures, pages 51-40, 2018.

[26] M Fares, Y Fargier, G Villain, X Dérobert, and S Palma-Lopes. Determining the permittivity profile inside reinforced concrete using capacitive probes. NDT \& E International, 79:150-161, 2016.

[27] K Vozoff and DLB Jupp. Joint Inversion of Geophysical Data. Geophysical Journal International, 42(3):977-991, sep 1975.

[28] E Haber and D Oldenburg. Joint inversion: a structural approach. Inverse Problems, 13(1):63-77, 1997.

[29] JA Doetsch, N Linde, and A Binley. Cross-gradients Joint Inversion of Time-lapse Crosshole ERT and GPR Data. In Near Surface 2010 - 16th European Meeting of Environmental and Engineering Geophysics 6 - 8 September 2010, Zurich, Switzerland, 2010.

[30] FO. Alpak, C Torres-Verdín, and TM Habashy. Joint inversion of transient-pressure and de resistivity measurements acquired with in-situ permanent sensors: A numerical study. Geophysics, 69(5):1173-1191, 2004

[31] LA Gallardo. Joint two-dimensional DC resistivity and seismic travel time inversion with cross-gradients constraints. Journal of Geophysical Research, 109(B3):1-11, 2004.

[32] E Kazatchenko, M Markov, and A Mousatov. Simulation of acoustic velocities, electrical and thermal conductivities using unified pore-structure model of double-porosity carbonate rocks. Journal of Applied Geophysics, 59(1):16-35, 2006.

[33] İ Demirci, ME Candansayar, A Vafidis, and P Soupios. Two dimensional joint inversion of direct current resistivity, radio-magnetotelluric and seismic refraction data: An application from Bafra Plain, Turkey. Journal of Applied Geophysics, 139:316-330, 2017.

[34] B Heincke, M Jegen, M Moorkamp, RW Hobbs, and J Chen. An adaptive coupling strategy for joint inversions that use petrophysical information as constraints. Journal of Applied Geophysics, 136:279-297, 2017.

[35] G Villain, A Ihamouten, and X Dérobert. Determination of concrete water content by coupling electromagnetic methods: Coaxial/cylindrical transition line with capacitive probes. NDT \& E International, 2017.

[36] ZM Sbartaï, V Garnier, G Villain, and D Breysse. 8 - Assessment of Concrete by a Combination of Non-Destructive Techniques. In Jean-Paul Balayssac, Vincent B T Non-Destructive Testing Garnier, and Evaluation of Civil Engineering Structures, editors, Non-Destructive Testing and Evaluation of Civil Engineering Structures, pages 259-297. Elsevier, 2018.

[37] JF Lataste. Presentation of common non destructive techniques, Electrical resistivity measurement. In D Breysse, editor, Non-Destructive Assessment of Concrete Structures: Reliability and Limits of Single and Combined Techniques, chapter 2, pages 77-85. Toulouse, 2012.

[38] F Wenner. A method for measuring Earth resistivity. Journal of the Washington Academy of Sciences, 5(16):561-563, 1915.

[39] L Marescot, S Rigobert, S Palma Lopes, R Lagabrielle, and D Chapellier. A general approach for DC apparent resistivity evaluation on arbitrarily shaped 3D structures. Journal of applied geophysics, 60(1):55-67, 2006.

[40] X Dérobert, J Iaquinta, G Klysz, and J.P. Balayssac. Use of capacitive and GPR techniques for the non-destructive evaluation of cover concrete. NDT \& E International, 41(1):44-52, 2008.

[41] KY Ann and HW Song. Chloride threshold level for corrosion of steel in concrete. Corrosion Science, 49(11):4113-4133, 2007.

[42] R Henkensiefken, J Castro, D Bentz, T Nantung, and J Weiss. Water absorption in internally cured mortar made with water-filled lightweight aggregate. Cement and Concrete Research, 2009.

[43] S Multon and F Toutlemonde. Effect of moisture conditions and transfers on alkali silica reaction damaged structures. Cement and Concrete Research, 2010.

[44] J Castro, D Bentz, and J Weiss. Effect of sample conditioning on the water absorption of concrete. Cement and Concrete Composites, 2011.

[45] S Poyet, S Charles, N Honoré, and V L'Hostis. Assessment of the unsaturated water transport properties of an old concrete: Determination of the pore-interaction factor. Cement and Concrete Research, 2011.

[46] RR Hussain, T Ishida, and M Wasim. Oxygen transport and corrosion of steel in concrete under varying concrete cover, w/c,and moisture. ACI Materials Journal, 109(1):3-10, 2012.

[47] F Ghasemzadeh, R Rashetnia, D Smyl, and M Pour-Ghaz. A comparison of methods to evaluate mass transport in damaged mortar. Cement and Concrete Composites, 70:119-129, 2016.

[48] GE Archie. The Electrical Resistivity Log as an Aid in Determining Some Reservoir Characteristics. Petroleum Transactions of the AIME, 146:54-62, 1942.

[49] Q Li, S Xu, and Q Zeng. The effect of water saturation degree on the electrical properties of cement-based porous material. Cement and Concrete Composites, 2016.

[50] M Vauhkonen, D Vadasz, PA Karjalainen, E Somersalo, and JP Kaipio. Tikhonov regularization and prior information in electrical impedance tomography. IEEE Transactions on Medical Imaging, 17(2):285-293, 1998.

[51] M Soleimani and WRB Lionheart. Nonlinear image reconstruction for electrical capacitance tomography using experimental data. Measurement Science and Technology, 16(10):1987-1996, 2005.

[52] W Jason, S Ken, B Jeff, and B Dale. Using a Saturation Function to Interpret the Electrical Properties of Partially Saturated Concrete. Journal 
Joint inversion of electromagnetic measurements for the determination of water saturation profiles in concrete structures

of Materials in Civil Engineering, 25(8):1097-1106, aug 2013.

[53] Y Fargier. Développement de l'Imagerie de Résistivité Électrique pour la reconnaissance et la surveillance des Ouvrages Hydrauliques en Terre. PhD thesis, 2011.

[54] LM Heikkinen, T Vilhunen, RM West, and M Vauhkonen. Simultaneous reconstruction of electrode contact impedances and internal electrical properties: II. Laboratory experiments. Measurement Science and Technology, 13(12):1855-1861, 2002.

[55] A Voss, M Pour-Ghaz, M Vauhkonen, and A Seppänen. Electrical capacitance tomography to monitor unsaturated moisture ingress in cement-based materials. Cement and Concrete Research, 2016.

[56] MR Silvester and RL Ferrari. Finite elements for electrical engineers. Cambridge University Press, 2nd edition, 1990.

[57] T Günther, C Rücker, and K Spitzer. Three-dimensional modelling and inversion of dc resistivity data incorporating topography — ii. inversion. Geophysical Journal International, 166(2):506-517, 082006.

[58] K Levenberg. A method for the solution of certain non-linear problems in least squares. Quarterly of Applied Mathematics, 2:164-168, 1944.

[59] D Marquardt. An algorithm for least-squares estimation of nonlinear parameters. SIAM Journal on Applied Mathematics, 11(2):431-441, 1963.

[60] PR Mcgillivray and DW Oldenburg. Methods for Calculating Fréchet for the Non-Linear Inverse Problem :. Geophysical Prospecting, (October 1989):499-524, 1990.

[61] MH Loke. 1-D Resistivity, IP \& SIP Inversion and forward modeling. Res1D ver. 1.00.09 Beta, 2001.

[62] J Goupy. Introduction aux Plans d'expériences. Paris, dunod edition, 2001.

[63] O Cazalla, E Sebastián, G Cultrone, M Nechar, and MG Bagur. Three-way ANOVA interaction analysis and ultrasonic testing to evaluate air lime mortars used in cultural heritage conservation projects. Cement and Concrete Research, 1999.

[64] A Soudki, F El-Salakawy, and B Elkum. Full Factorial Optimization of Concrete Mix Design for Hot Climates. Journal of Materials in Civil Engineering, 13(6):427-433, dec 2001.

[65] LM Del Río, A Jiménez, F López, FJ Rosa, MM Rufo, and JM Paniagua. Characterization and hardening of concrete with ultrasonic testing. In Ultrasonics, 2004.

[66] G Villain and M Thiery. Gammadensimetry: Amethod to determine drying and carbonation profiles in concrete. NDT \& E International, 39(4):328-337, 2006. 